\title{
Search for diffuse gamma-ray emission from the Galactic plane with IceCube
}

\author{
The IceCube Collaboration ${ }^{\dagger}$ \\ $\dagger$ http://icecube.wisc.edu/collaboration/authors/icrc17_icecube \\ E-mail: hershaleudel .edu
}

We present a search for diffuse $\mathrm{PeV}$ gamma-rays arising from interactions of cosmic rays with the interstellar gas in the Galactic plane. We analyze air shower data recorded by the IceCube Observatory from May 2011 to May 2016 in the energy range of $0.6 \mathrm{PeV}$ to $100 \mathrm{PeV}$ for the presence of gamma-ray showers. Muon poor gamma-ray induced air showers are discriminated from the cosmic ray showers using the characteristics of signals recorded by IceTop as well as the in-ice IceCube array. We carry out an unbinned maximum likelihood analysis on the Galactic plane region $\left(270^{\circ} \lesssim l \lesssim 335^{\circ}\right)$ located in the IceCube field of view. The pion decay component of the Fermi-LAT diffuse emission model is used as the spatial template for the analysis. No statistically significant evidence for diffuse gamma-ray emission was found. We derive an upper limit of $1.04 \times 10^{-9} \mathrm{GeV} \mathrm{cm}^{-2} \mathrm{~s}^{-1}$ (90\% confidence limit) on the normalization of the spectral energy distribution at $2 \mathrm{PeV}$ assuming an $\mathrm{E}^{-3}$ spectrum.

Corresponding authors: Hershal Pandya ${ }^{* 1}$, Zachary Griffith ${ }^{2}$

${ }^{1}$ Dept. of Physics and Astronomy, University of Delaware, Newark, DE 19711, USA.

${ }^{2}$ Wisconsin IceCube Particle Astrophysics Center (WIPAC) and Department of Physics, University of Wisconsin-Madison, Madison, WI 53706, USA.

35th International Cosmic Ray Conference - ICRC2017

10-20 July, 2017

Bexco, Busan, Korea

${ }^{*}$ Speaker. 


\section{Introduction}

Cosmic ray interactions with the interstellar gas produce neutral pions that further decay into gamma-rays. Hence, high energy gamma-rays can be used for measuring the spectral density of the Galactic cosmic rays and probing their propagation. Diffuse gamma-ray emission from the Galactic plane has been measured up to $\mathrm{TeV}$ energies but it has not been observed in the $\mathrm{PeV}$ energy range. Along with the significant decrease in their flux as compared to $\mathrm{TeV}$ gamma-rays, there is yet another process that makes the observation of $\mathrm{PeV}$ gamma-rays challenging. PeV gamma-rays are significantly attenuated by interactions with the cosmic microwave background radiation. This limits the observable source distance to few tens of kiloparsecs [1] and hence within the Galaxy. The same cosmic ray interactions that are responsible for producing neutral pions, also produce charged pions that decay into neutrinos. Therefore, measurement of diffuse gamma-rays could also provide insight into a Galactic origin for some of the astrophysical neutrinos observed by IceCube $[2,3]$.

$\mathrm{PeV}$ gamma-rays can be detected via the extensive air showers of particles generated from their interaction with nuclei in the Earth's atmosphere. Air showers generated by photon primaries differ from cosmic ray air showers in their longitudinal shower development (shower age) as well as the muon content. Based on showers simulated using CORSIKA [4] with hadronic interaction models FLUKA [5] and SIBYLL 2.1 [6], cosmic ray showers have roughly ten times the number of $\mathrm{GeV}$ muons as compared to gamma-ray showers, whereas for muons with energy greater than $100 \mathrm{GeV}$ this ratio increases to about a hundred. Photon showers are also younger since their shower maximum occurs deeper in the atmosphere than the cosmic ray showers. We use IceCube to measure these properties and discriminate gamma-ray showers from the highly abundant cosmic ray showers.

In search of diffuse gamma-ray emission from the Galactic plane, we carry out an unbinned maximum likelihood analysis. We improve upper limits on diffuse PeV gamma-ray emission at Galactic longitudes $270^{\circ} \lesssim l \lesssim 335^{\circ}$ by one order of magnitude.

\section{Detector}

IceCube [7], as shown in Fig. 1, is a cubic kilometer array of 5160 optical sensors arranged on 86 strings, embedded in the Antarctic ice at a depth between $1450 \mathrm{~m}$ to $2450 \mathrm{~m}$ under the surface. The strings have a horizontal spacing of $125 \mathrm{~m}$ with the optical sensors vertically separated by $17 \mathrm{~m}$ on each string. The optical sensors, digital optical modules (DOMs), detect Cherenkov radiation emitted by relativistic charged particles traversing the ice. The charged particles may be downward-going high energy muons from cosmic ray air showers or from neutrino interactions in the surrounding ice or the bedrock. IceCube has an additional component called IceTop, which is located on the surface at an altitude of $2835 \mathrm{~m}$ above sea level and triggers on extensive air showers. IceTop is sensitive to air showers from cosmic rays with primary energies in the range of $300 \mathrm{TeV}$ to $1 \mathrm{EeV}$. It consists of 81 pairs of ice tanks that cover an area of about one square kilometer, with two DOMs within each tank. IceTop DOMs are calibrated using the vertical muons from low energy air showers and the signals are measured in units of vertical equivalent muons (VEM). The direction of the primary particle, the shower size, and the location of the shower core 
on the surface, are reconstructed by simultaneously fitting a lateral distribution function (LDF) to measured charge depositions, and a shower curvature function to the signal times. The lateral distribution function, describing the signal distribution as a function of the lateral distance from the shower axis, is defined as

$$
S(R)=S_{125}\left(\frac{R}{125 m}\right)^{-\beta-0.303 \log _{10}\left(\frac{R}{125 m}\right)}
$$

where $S(R)$ is the signal measured at a lateral distance $R$ from the shower axis, $\beta$ is the slope of the logarithmic LDF at $125 \mathrm{~m}$, and the signal measured at $125 \mathrm{~m}, S_{125}$, is the shower size.

The energy of the primary particle is calculated by using a $\log _{10}\left(S_{125}\right)$ to $\log _{10}(E)$ conversion obtained from simulated cosmic ray showers [8]. Snow accumulates on top of the IceTop tanks with time, and attenuation of the signal due to snow is accounted for while reconstructing the shower size [9].

\section{Analysis}

The current analysis can be divided into two parts: first an enriched sample of gamma-ray like events from the observed data is obtained. In a second step, this enriched sample is used for a maximum likelihood analysis to search for diffuse emission from the Galactic plane. We analyze air shower events recorded by IceCube between May

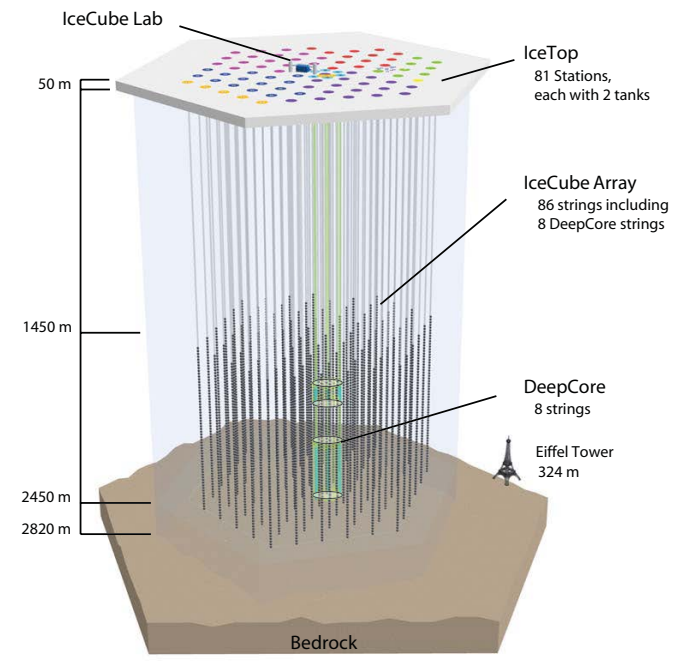

Figure 1: The IceCube Detector with its components DeepCore and IceTop. 2011 and May 2016 with sufficient energy to trigger ten IceTop tanks or more. For identifying gamma-ray air showers, we rely on simulated showers generated using CORSIKA [4] with low- and high-energy hadronic interaction models FLUKA [5] and SIBYLL 2.1 [6], respectively. The detector response to gamma-ray showers was simulated for each of the five years with different snow heights on top of the tanks as recorded during October or November of each year. To avoid any bias and to maintain blindness to the source, the subsequent event selection as well as the maximum likelihood analysis was developed using only $10 \%$ of the available data. Various quality cuts were placed on both data as well as simulations to obtain a sample of well reconstructed air showers.

\subsection{Event Selection}

Using IceTop observables, we construct three two-dimensional probability distribution functions (PDFs) that incorporate different shower characteristics. For example, Fig. 2 shows the two-dimensional PDF constructed using the measured charge in tanks, and their lateral distance from the shower axis. This PDF represents the lateral distribution of charges. One of the important features in this PDF is the $\sim 1$ VEM signal due to $\mathrm{GeV}$ muons emerging at large lateral distances for cosmic ray showers (highlighted using dashed lines in Fig. 2). This feature is absent for the 

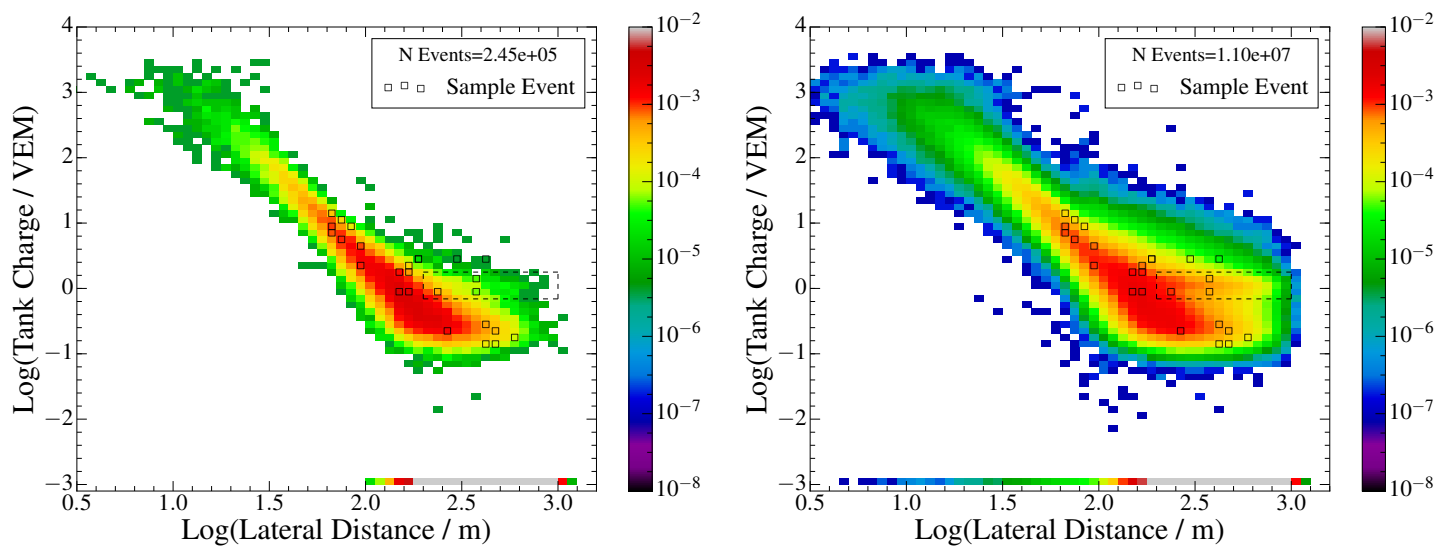

Figure 2: PDFs for photon (left) and cosmic ray (right) events, based on lateral distribution of charges, with $0.3 \leq \log _{10}\left(S_{125}\right)<0.4$ and $0.9 \leq \cos (\theta)<0.95$. Hit tanks for a typical cosmic-ray event are shown as hollow boxes and the signal due to $\mathrm{GeV}$ muons is highlighted using dashed lines.

gamma-ray showers. The likelihood defined as the product of probabilities for all tanks, is computed for each event, using either a gamma-ray or a cosmic ray shower PDF. A log-likelihood ratio is the difference of log-likelihoods obtained from gamma-ray and cosmic ray PDFs. A typical cosmic-ray event overlaid on the PDFs in Fig. 2, will have a large log-likelihood ratio due to contribution from the muon feature at large distances. Other features such as the steepness and thickness of the LDF, which correlate with the shower age and hadronic content, also contribute to the log-likelihood ratio. We calculate two more log-likelihood ratios for every event, from PDFs based on the time distribution of charges and the shower front shape [10]. All three ratios are added to form a single variable, IceTop LLH ratio, used in the final event selection.

For coincident events with the reconstructed axis passing through the in-ice detector, the measurement of IceCube charges provides a strong handle on the number of high energy muons. High energy muons $\left(\mathrm{E}_{\mu}>300 \mathrm{GeV}\right)$ can reach deep inside the ice and deposit energy in DOMs via radiation from ionization losses, stochastic losses, as well as direct Cherenkov radiation. To estimate the energy deposited by muons, cleaned in-ice charge is computed by adding charges from selected DOMs. The selection of DOMs is optimized to remove hits uncorrelated to air shower muons. The quality of separation from both, IceTop LLH ratio and cleaned in-ice charges, increases with increasing shower size (primary energy), as shown in Fig. 3.

Events with reconstructed zenith angle $0^{\circ}<\theta<37^{\circ}$, and reconstructed energy between 0.6 $\mathrm{PeV}$ and $100 \mathrm{PeV}$, are included in the current analysis. The event selection was done by using a random forest algorithm trained to discriminate gamma-ray events from cosmic ray events. The machine learning algorithm was implemented using the open source python package scikit-learn [11]. The random forest was trained using the following features: IceTop LLH ratio, cleaned in-ice charge, $S_{125}$, zenith angle, and a measure of geometric containment of the shower track in IceCube. Gamma-ray events used for training were weighted according to an $\mathrm{E}^{-3}$ spectrum. Finally, signal and background events with a classifier score above a cut value were used for the maximum likelihood analysis. Of all the selected events, $5 \%$ of the events are below $0.68 \mathrm{PeV}$, 

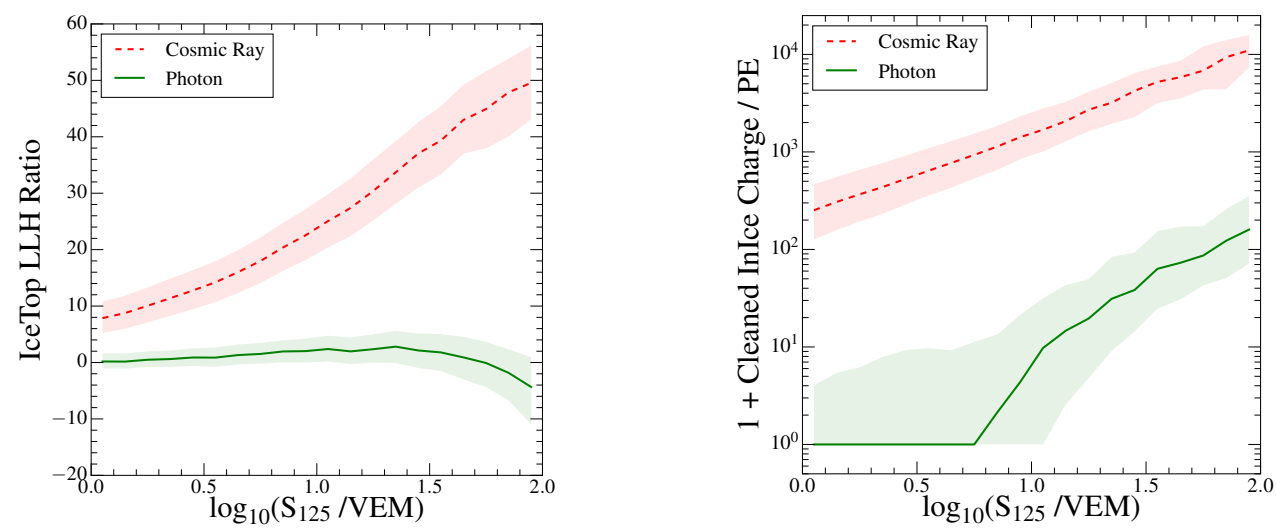

Figure 3: IceTop LLH ratio as a function of $\log _{10}\left(S_{125}\right)$ for all events (left). Cleaned in-ice charge as a function of $\log _{10}\left(S_{125}\right)$ for coincident events (right). Shaded regions delimit $50 \%$ of the distribution around the median.

and $95 \%$ of the events are below $2.73 \mathrm{PeV}$. Roughly $0.2 \%$ of the background events pass the cut between $0.68 \mathrm{PeV}$ and $2.73 \mathrm{PeV}$ while retaining $57 \%$ of the signal events.

\subsection{Unbinned Maximum Likelihood Analysis}

We implement a modified version of the usual unbinned likelihood analysis [12] that utilizes a spatial template for the expected diffuse signal and depends on constructing the background hypothesis from observed data. The usual likelihood function is defined as

$$
L\left(n_{s}, \gamma\right)=\prod_{i=1}^{N}\left(\frac{n_{s}}{N} S_{i}\left(\mathbf{x}_{i}, \sigma_{i}, E_{i} ; \gamma\right)+\left(1-\frac{n_{s}}{N}\right) B_{i}\left(\sin \delta_{i}, E_{i}\right)\right),
$$

where $n_{s}$ is the number of signal events for a flux following spectral index $\gamma ; N$ is the total number of events in the sample; $S_{i}$ is the signal PDF for the $i^{t h}$ event, located at sky coordinates $\mathbf{x}_{i}=\left(\alpha_{i}, \delta_{i}\right)$, with energy $E_{i}$, and angular resolution $\sigma_{i}$. The background PDF $B_{i}$, for declination $\delta_{i}$ and energy $E_{i}$, is usually approximated by the event density of the real data integrated over right ascension. But unlike a point source, the signal from the Galactic plane may extend over the entire field of view. Hence for the assumption where $n_{s}>0$, the signal present in the event density of the real data is not negligible. Thus, a modified likelihood function employing the signal-subtracted background is given by,

$$
L\left(n_{s}, \gamma\right)=\prod_{i=1}^{N}\left(\frac{n_{s}}{N} S_{i}\left(\mathbf{x}_{i}, \sigma_{i}, E_{i} ; \gamma\right)+\widetilde{D}_{i}\left(\sin \delta_{i}, E_{i}\right)-\frac{n_{s}}{N} \widetilde{S}_{i}\left(\sin \delta_{i}, E_{i}\right)\right)
$$

where $\widetilde{D}$ and $\widetilde{S}$ are the event densities of the real data and simulated signal, respectively, integrated over right ascension. The signal PDF is constructed from the $\pi^{0}$ decay template (Fig. 4a) of the Fermi-LAT diffuse emission model [13]. To obtain a true signal PDF as it would be observed by IceCube (Fig. 4b), the spatial template is multiplied by the detector's acceptance to gamma-rays. The angular uncertainty of the events is incorporated in the likelihood by convolving the map with the point spread function (PSF) of the event, which is described by a Gaussian distribution of width $\sigma$ (Fig. 4c). 


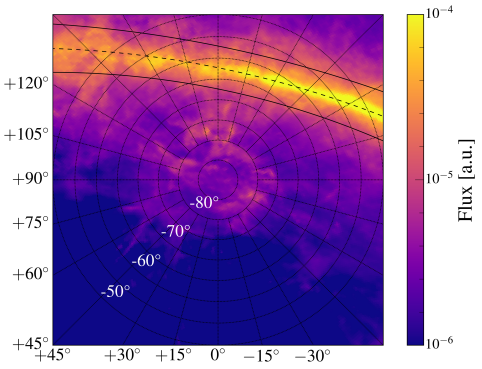

(a) Fermi-LAT $\pi^{0}$ decay template.

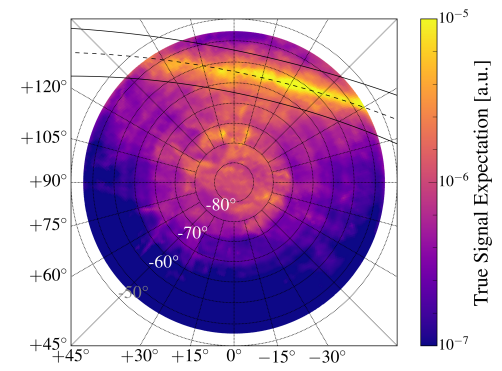

(b) Signal PDF in true coordinates

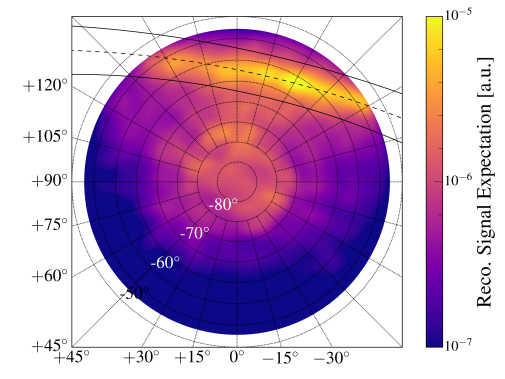

(c) Signal PDF in reconstructed coordinates for $1.0^{\circ} \mathrm{PSF}$.

Figure 4: Steps for construction of signal PDF. Maps are plotted in equatorial coordinates and the dashed line shows the Galactic plane along with $|b|<5$ bounds as solid lines.

The test statistic is then defined as the log-likelihood ratio of the best-fit signal strength and the null hypothesis, which corresponds to no Galactic diffuse emission. We have validated that the test statistic distribution for the null hypothesis $n_{s}=0$ follows a $\chi^{2}$-distribution with one degree of freedom. The template analysis yields an angular-integrated flux $\phi^{\prime}$ from the diffuse source region within the field of view. The spectral energy distribution defined as the flux scaled by $\mathrm{E}^{2}$, is given by the equation

$$
E^{2} \phi^{\prime}(E)=A\left(\frac{E}{E_{0}}\right)^{2-\gamma}
$$

where $\gamma$ is the assumed source spectral index, $A$ is the normalization constant, and $E_{0}$ is the reference energy. The median upper limit, as reported in Sec. 4, is the normalization $A$ corresponding to a signal flux $\phi^{\prime}(E)$ that generates a test statistic distribution with $90 \%$ of trials above the median of the null hypothesis distribution.

\subsection{Spectral Index Assumption}

Uncertainties in the Galactic cosmic ray spectrum, interstellar gas distribution, and flux attenuation between the source and the observer, result in an uncertainty in the observed spectral index for the diffuse emission. Calculations predict the unattenuated flux in the PeV energy range to follow a spectrum as hard as $\mathrm{E}^{-3}[14,15]$, and the spectrum could be as soft as $E^{-3.4}$ [15] for the attenuated flux. Hence it becomes important to investigate the effect of the choice of spectral index on our analysis. We carry out the maximum likelihood analysis with various spectral index assumptions with the results shown in Fig. 3.2. The analysis is least sensitive to the spectral index assumption at about $2 \mathrm{PeV}$. Hence we quote the final median upper limit on the normalization $A$ for $E_{0}=2 \mathrm{PeV}$ and $\gamma=3$.

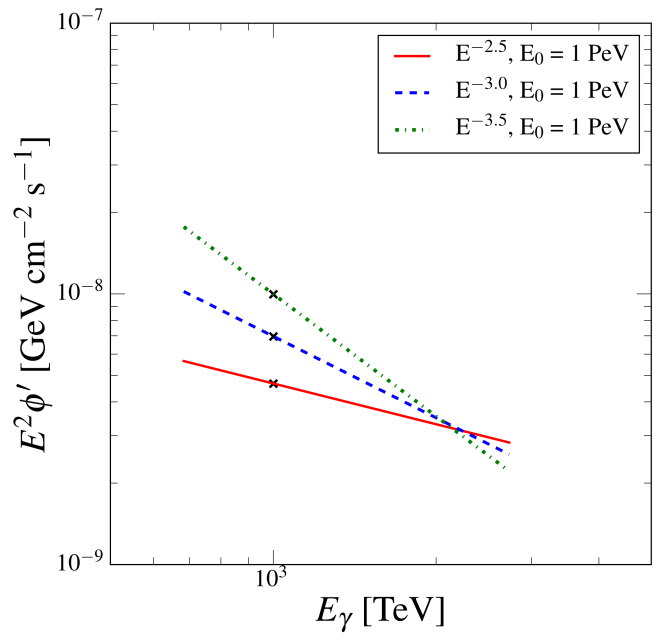

Figure 5: $90 \%$ C.L. upper limit spectral energy distribution with normalization at $1 \mathrm{PeV}$ assuming different spectral indices. 


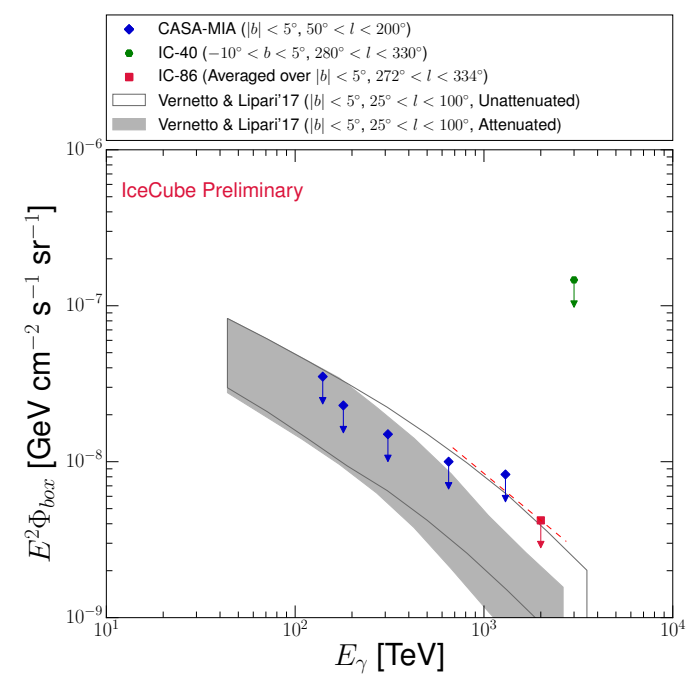

(a) Flux from a boxed region around the Galactic plane. For IC-86, it is an average flux from the boxed region.

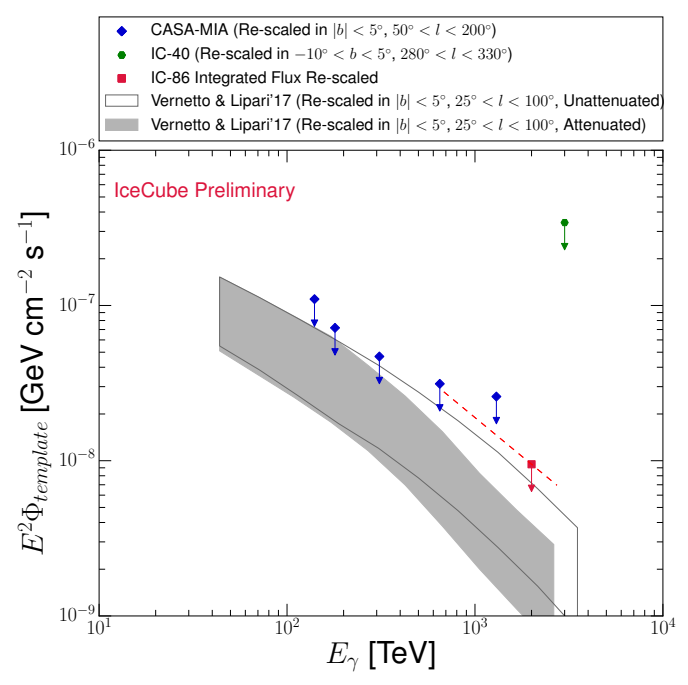

(b) Angular-integrated flux rescaled by the observed Galactic plane fraction calculated using the Fermi template.

Figure 6: IceCube 90\% C.L. upper limit on the diffuse gamma-ray emission from the Galactic plane in our FOV as compared with the previous IceCube analysis using a partial detector IC-40 [16] and CASA-MIA [17]. The result from this analysis was obtained by using the Fermi-LAT $\pi^{0}$ decay spatial template [13] as compared to IC-40 and CASA-MIA which define a boxed region around the Galactic plane. Dotted lines show the $\mathrm{E}^{-3}$ spectrum, used for obtaining IceCube upper limits, over the energy range containing $5 \%$ to $95 \%$ events in the final sample. Also shown are unattenuated and attenuated flux predictions from Ref. [14].

\section{Results And Discussion}

We find no significant evidence for diffuse PeV gamma-rays from the Galactic plane. The observed test statistic corresponds to a p-value of $20.1 \%$, and hence we do not exclude the null hypothesis of no diffuse emission. We place a $90 \%$ confidence level upper limit of $1.04 \times 10^{-9}$ $\mathrm{GeV} \mathrm{cm}^{-1} \mathrm{~s}^{-1}$ on the normalization of the spectral energy distribution described in Eq. 3.3 for $E_{0}=2 \mathrm{PeV}$ and $\gamma=3$. We have yet to gauge the dependence of current results on the choice of spatial template.

Typically, results of $\mathrm{TeV}-\mathrm{PeV}$ gamma-ray emission along the Galactic plane are quoted as a diffuse flux within a box-shaped region in latitude $b$ and longitude $l$. In order to compare our template based result to these observations, we use the following procedures. At first, we compare the flux from a boxed region $\left(\Phi_{b o x}\right)$ around the Galactic plane (Fig. 6a). To calculate the approximate $\Phi_{b o x}$ for this analysis, we use the Fermi template to find the fraction of the angular-integrated flux from this region: $\left(|b|<5^{\circ}, 272^{\circ}<l<334^{\circ}\right)$; and divide it by the corresponding solid angle. For the second comparison (Fig. 6b), we propose an angular-integrated scaled flux as

$$
\Phi_{\text {template }}=\Phi \Delta \Omega \frac{\int_{\text {all sky }} S_{\text {Fermi }} d \Omega}{\int_{\Delta \Omega} S_{\text {Fermi }} d \Omega},
$$


where $\Phi \Delta \Omega$ gives the angular-integrated flux from the observed region, and the second term scales this flux by the fraction of Galactic plane, present in the observed region, as given by the Fermi template $S_{\text {Fermi }}$. In general, a template based analysis provides a more conservative upper limit than the analysis performed with the assumption of uniform emission from a boxed region.

In summary, the emission of PeV gamma-rays from the Galactic plane provides a measure of the Galactic distribution of cosmic rays in the knee region. The observation can provide valuable information for the modeling of cosmic ray propagation in, and escape from, our Galaxy (e.g., Ref. [18]). Our results complement the other strong limits derived by CASA-MIA, by placing competitive upper limits on the flux from a distinct portion of the Galactic plane. Result from this analysis corresponds to the strongest upper limit in the Southern Hemisphere, improving the previous limits by one order of magnitude.

\section{References}

[1] S. Vernetto and P. Lipari, Phys. Rev. D. 94 (2016) 063009.

[2] IceCube Collaboration, M. G. Aartsen et al., Phys. Rev. Lett. 113 (2014) 101101.

[3] M. Ahlers and K. Murase., Phys. Rev. D 90 (2014) 023010.

[4] D. Heck, G. Schatz, T. Thouw, J. Knapp, and J. N. Capdevielle, Report No. FZKA 6019 (1998) .

[5] A. Ferrari, P.R. Sala, A. Fasso, and J. Ranft, CERN-2005-10 (2005), INFN/TC_05/11, SLAC-R-773.

[6] E. J. Ahn, R. Engel, T. K. Gaisser, P. Lipari, and T. Stanev , Phys. Rev. D 80 (2009) 094003.

[7] IceCube Collaboration, Aartsen et al., J. Inst. 12 (2017) P03012.

[8] IceCube Collaboration, T. Feusels, PoS ( ICRC2015) 334 (2015).

[9] IceCube Collaboration, R. Abbasi et al., Nucl. Instrum. Meth. A 700 (2013) 188.

[10] IceCube Collaboration, H. Pandya et al., PoS (ICRC2017) 514 (these proceedings).

[11] F. Pedregosa et al., J. Mach. Learn. Res. 12 (2011) 2825.

[12] IceCube Collaboration, C. Haack et al., PoS ( ICRC2 017) 1011 (these proceedings).

[13] M. Ackermann et al., Astrophys. J. 750 (2012) 3.

[14] S. Vernetto and P. Lipari, EPJ Web of Conf. 136 (2017) 03015.

[15] G. Ingleman and M. Thunman, arXiv (1996) hep-ph/9604286.

[16] IceCube Collaboration, M. G. Aartsen et al., Phys. Rev. D 87 (2013) 062002.

[17] A. Borione et al., Astrophys. J. 493 (1998) 175.

[18] D. Gaggero et al., Astrophys. J. Lett. 815 (2015) L25. 